\title{
Contra el despojo total. Perspectivas de una experiencia de acción social en Caño Negro, Costa Rica
}

\author{
Against total dispossession. Perspectives of a social action \\ experience in Caño Negro, Costa Rica
}

Daniel Torres Sandí ${ }^{1}$

Juan Manuel Retana Céspedes ${ }^{2}$

Lea Audibert González ${ }^{3}$

Lidia Peña Rivera ${ }^{4}$

Luis Diego Arias Campos 5

\footnotetext{
${ }^{1}$ Universidad de Costa Rica. Construcción Organizativa Socio Ambiental (COSA). Costa Rica. Correo electrónico: tsdaniel22@hotmail.com

${ }^{2}$ Universidad de Costa Rica. Construcción Organizativa Socio Ambiental (COSA). Costa Rica. Correo electrónico:jmretanac@gmail.com

${ }^{3}$ Universidad de Costa Rica. Construcción Organizativa Socio Ambiental (COSA). Costa Rica. Correo electrónico: leaudigo@gmail.com

${ }^{4}$ Universidad de Costa Rica. Construcción Organizativa Socio Ambiental (COSA). Costa Rica. Correo electrónico: liberlu09@gmail.com

${ }^{5}$ Universidad de Costa Rica. Construcción Organizativa Socio Ambiental (COSA). Costa Rica. Correo electrónico: luisdiegoarias93@gmail.com
} 
Resumen: En este manuscrito se plantea, a través de la experiencia en Caño Negro (específicamente El Sitio y Las Flores), perspectivas que construyan formas de convivencia que permitan el buen vivir. La experiencia en esta comunidad refleja la organización comunitaria, aunado a la educación y la agricultura, como bases fundamentales para plantear formas de subsistencia alternativas al modelo agroindustrial impuesto en la región, permeado por la explotación laboral y la destrucción ecológica. Por lo tanto, es necesario resaltar y revitalizar los beneficios ecosociales de la vida en el campo ante las presiones y amenazas de parte de la agroindustria, y hacerla atractiva y sostenible en el tiempo.

Palabras clave: agroindustria, organización comunitaria, colapso ecológico, agricultura.

Abstract: In this manuscript, through the experience in Caño Negro (specifically El Sitio and Las Flores), perspectives are proposed that build forms of coexistence that allow good living. The experience in this community reflects the community organization, together with education and agriculture, as fundamental bases for proposing alternative forms of subsistence to the agro-industrial model imposed in the region, permeated by labor exploitation and ecological destruction. Therefore, it is necessary to highlight and revitalize the ecosocial benefits of life in the countryside in the face of pressures and threats from the agro-industry, and make it attractive and sustainable over time.

Key Words: agroindustry, community organization, ecological collapse, agriculture.

\section{Introducción}

La motivación de este manuscrito surge de la experiencia del colectivo estudiantil Construcción Organizativa Socio Ambiental (Cosa), con el propósito de acompañar la experiencia de organización comunitaria y prácticas agroecológicas que enriquezcan la salud física y mental, la educación, la amistad, etc., ante la degradación ecosocial provocada por la reconversión productiva y política que por casi 30 años ha devastado los ecosistemas, ha presionado la desposesión de la tierra, ha empobrecido a las poblaciones y las ha sometido a condiciones de explotación física y psicológica. 
Este ensayo pretende reflexionar sobre la experiencia de acción social interdisciplinaria que se ha implementado por parte del colectivo Cosa en una comunidad rural de Caño Negro, en la Región Huetar Norte, como formas de adaptación al contexto agroindustrial y la vulneración de la vida en el campo, a través del acompañamiento de investigación-acción-participante que enriquezca la construcción de comunidades organizadas en reacción a los modelos extractivistas.

A manera de estructura, se comienza explorando el engranaje compuesto por la producción agroindustrial controlado por medianas y grandes compañías multinacionales (y nacionales), para luego abordar el tema del abandono de la tierra como una consecuencia estructural de la acumulación espacial y la degradación de la vida campesina. Esto permite contextualizar la situación de Caño Negro como una comunidad inmersa las dinámicas desiguales de la distribución de la riqueza y el espacio, abordando la experiencia en la comunidad de El Sitio y Las Flores.

\section{La situación de los monocultivos}

Con despojo total hacemos referencia no solo al despojo del espacio (tierra), sino también al despojo económico, social, cultural, ideológico, ético, ambiental y de recursos vitales a través de la explotación laboral, el agotamiento físico y psicológico, la deforestación, la contaminación de las aguas, del suelo, de los mantos acuíferos, del aire, etc., que intensifica las enfermedades, las plagas y el malestar, tal como lo demuestran estudios como el de Carazo \& Aravena (2016) para la organización Oxfam en la producción industrial piñera.

Las condiciones de colapso ontológico ecosocial (Saxe Fernández 2005) que está enfrentando la humanidad responde a diversos factores, entre los que se encuentran las malas prácticas en la agroindustria, la ganadería extensiva, la sobreexplotación de recursos, entre otros; que han desencadenado una serie de situaciones climáticas, económicas, políticas y sociales que se han intensificado con la implementación de los Programas de Ajuste Estructural (Pae) y tratados de libre comercio para la consolidación del modelo neoliberal que han tenido como bandera principalmente la reducción de los Estados, y la intensificación de las plusvalías direccionadas al sector privado (Petras \& Veltmeyer 2002). 
Los privilegios a las multinacionales se sitúan más que todo a partir de los ochenta del siglo XX, cuando el expresidente Óscar Arias Sánchez (1986-1990) promulgó la "Agricultura del Cambio" (Llaguno Thomas et al. 2014). Los préstamos Banco Mundial para salir de la crisis de esa década estaban condicionados a las imposiciones del Fondo Monetario Internacional (FMI) para instaurar su control hegemónico y la imposición de los poderes económicos imperiales sobre América Latina, recortando préstamos y apoyos en el sector rural (Petras \& Veltmeyer 2002).

Además de la tarea de efectuar el 'drenado de recursos desde el campo' para cumplir con las obligaciones de la deuda externa (...), (existen) otros papeles le son asignados al Estado colonizado en América Latina: Con miras a ejercer la vigilancia policiaca sobre los campesinos desplazados y los trabajadores rurales desocupados, los Estados colonizados desnacionalizan la propiedad de la tierra y privatizan las empresas rentables de los sectores clave de la economía (Petras \& Veltmeyer 2002: 34. Énfasis propio sin cursivas).

Los monocultivos a gran escala no han sido capaces de erradicar el hambre como lo prometía la Revolución Verde posterior a la Segunda Guerra Mundial, y por el contrario, más bien ha dificultado la vida en el campo. Eso lo demuestra por ejemplo la hambruna que sufrieron 795 millones de personas en 2015 alrededor del mundo (Fao 2015). De hecho, los enormes daños ecosociales de la agroindustria ha llevado a las mismas Naciones Unidas a repensar la forma de alimentar al mundo a través de la agricultura tradicional (United Nations 2013).

Ante el despojo, criminalización y asesinatos impunes de ambientalistas, indígenas y campesinos en resistencia, surge la necesidad ya ni siquiera de lograr mejoras económicas, sino de algo más urgente; garantizar la seguridad alimentaria y la vida misma (Alonso Reynoso \& Alonso Sánchez 2015). América Latina se ha vuelto la región del mundo que posee la distribución más desigual de la tierra (Guereña 2016), y en la más peligrosa para defender la naturaleza (Global Witness 2016), ya que el desarrollismo ve como obstáculo los derechos humanos y la resistencia como verdad ética (Comité Invisible 2015). Además, ven al Estado como un intermediario legitimador del saqueo, y como -más bien- un enemigo de las comunidades (Llaguno Thomas et al. 2014). ${ }^{6}$

\footnotetext{
6. Ver el Environmental Justice Atlas (https://ejatlas.org/) para tener una idea de la magnitud de los conflictos.
} 
Estas resistencias se vuelven fundamentales ante la imposición de paquetes agroindustriales extractivistas, así como la riesgosa y cada vez más inevitable dependencia al libre mercado para alimentarse, que más allá de abaratar los costos de los productos, intensifica la sobreproducción, el consumismo y la esclavitud moderna en las zonas de origen (Rosset 2009). Según Black (2013), resulta paradójico que la invención de máquinas y alucinantes tecnologías no ha logrado paliar la situación de explotación laboral y degradación ambiental que se viven en la ruralidad latinoamericana.

La tecnificación, capitalización y dominación de todo, hasta del ser, de la naturaleza y de las personas (Bookchin 1999), ha naturalizado la percepción de medir el valor de las cosas y la calidad de vida únicamente mediante parámetros econométricos llamado Capital Humano (Quesada Monge 2015). Pero la dignidad va más allá de la satisfacción material capitalista o la ausencia de enfermedades, sino que depende también del bienestar físico, mental y social (Baldí López \& García Quiroga 2005).

Quienes intentan una autonomía agrícola -no individualista- deben enfrentar un factor clave en la agricultura contemporánea; se trata de los efectos del colapso climático antropogénico, el cual está generando enormes pérdidas de cosechas por las inesperadas sequías, tormentas, proliferación de plagas, y el incierto abastecimiento alimentario (Artieri \& Toledo 2010). La seguridad alimentaria se encuentra en riesgo, sobre todo a partir de finales del siglo XX, cuando, para el caso de Costa Rica la estructura agroexportadora pasó de ser fundamentalmente de productos tradicionales (de $71.8 \%$ en los setenta, a $12 \%$ en 2011 ) a no tradicionales (6.6\% en los 1970, a 87.8\% en 2011) (Llaguno Thomas et al. 2014).

Algunas prácticas agrícolas abogan por resolver estos efectos climáticos principalmente con paquetes tecnológicos. De esta forma, no sólo intensifican su productividad, sino que también intentan resolver otras situaciones propias de los monocultivos mediante el uso intensivo de agrotóxicos, los cuales se calcula que provocan la muerte de unas 200 mil personas anualmente (Consejo de Derechos Humanos 2017). A pesar de eso, estas corporaciones también han logrado apropiarse de otros recursos como el agua, a veces por encima del consumo humano o local (ver por ejemplo Córdoba \& Rodríguez 0306-2015). 
El uso de agrotóxicos ha aumentado de manera preocupante, sobre todo en Costa Rica. En junio de 2015, un reportaje del Semanario Universidad, dos días antes de la conmemoración del Día Mundial del Medio Ambiente, informaba que Costa Rica alcanzó el primer lugar en consumo de agrotóxicos en el mundo (Araya 03-06-2015), un puesto que ha venido relevando con China. La globalización económica, y el reforzamiento del neoliberalismo como referente sociopolítico e identitario, ha provocado importantes transformaciones en las concepciones culturales alrededor de la agricultura, ante lo cual atendemos, por un lado, el olvido de prácticas agrícolas tradicionales de siembra y manejo natural de plagas, y por otro, un incremento de la dependencia a insumos como semillas, agrotóxicos, abonos, etc., suministrados por cooperativas y grandes corporaciones (Artieri \& Toledo 2010).

La Revolución Verde -a pesar de sus consecuencias- no solo hizo pensar a la humanidad que la mejor forma de producir era a través de la industrialización agrícola, sino que generó grandes destrucciones, y ha dejado como herencia corporaciones como Monsanto, Novartis, AgrEvo, DuPont y otras biotecnológicas que están reinventando la Revolución Verde, además del gran capital bancario (como el Banco Mundial) interviniendo sobre la agricultura mundial, y dedicadas a producir especies transgénicas, agrotóxicos, fármacos y demás productos de dependencia estratégica (Artieri \& Toledo 2010).

\subsection{El abandono de la tierra}

En América Latina existe un sector campesino con una población estimada en 65 millones, que incluye 40-55 millones de indígenas que hablan aproximadamente 725 idiomas (Toledo et al. 2010). Algunas estimaciones han calculado que estos campesinos producen el $51 \%$ del maíz, el 77\% de los granos y el 61\% de las papas que se consume en la región (Altieri 1999). Contrario a eso, se calcula que unos 500 millones de campesinos no tienen tierra en el mundo (Farah \& Vasapollo 2011).

Sumado a las consecuencias de la monocultivización y del libre comercio neoliberal, existe otro problema estructural que afecta a las poblaciones rurales, y que es tratado por el revelador y didáctico trabajo de Armando Quesada Webb (21-06-2017) para Costa Rica. Se trata de la descampesinización ante el colapso de las economías locales y el no relevo 
generacional. La instalación de oligo y monopolios agrícolas multinacionales ha provocado la precarización de las condiciones socioeconómicas de las regiones, llevando a los terratenientes locales a vender o abandonar sus tierras para migrar.

Este colapso de las economías locales ocurre ante el empobrecimiento de los pequeños comerciantes, por el abaratamiento de los productos provenientes del libre mercado internacional (Quesada Webb 21- 06-2017). Décadas antes de la implementación de los ajustes estructurales, los países centroamericanos habían logrado casi autosuficiencia en granos como arroz, frijoles, maíz y sorgo (Llaguno Thomas et al. 2014). Sin embargo, la transformación productiva ha llevado incluso a que los campesinos vean con desconfianza al Estado.

La migración campo-ciudad genera dependencias económicas que las personas rurales antes no poseían. Este importante traslado poblacional contribuyó a la consolidación de grandes anillos de urbano marginalidad en la mayoría de centros urbanos latinoamericanos. Esta escasez económica, surgida de la imposibilidad de las estructuras productivas urbanas de incorporar satisfactoriamente al contingente rural migrante, se une las crecientes dificultades de convivencia urbana armónica, la violencia y el narcotráfico.

Para todo aquel que no pueda contar con el apoyo de una familia u otro grupo mejor parado, la opción es sencilla: hundirse en la decadencia moral y física o acudir a medios ilícitos de obtención de recursos, lo que atrae sobre sí y justifica cada vez más la presión de las fuerzas de control y del encarcelamiento (Black 2013: 53).

En la degradación (o no) física y psicológica influye también las condiciones ambientales de paisajismo, aire puro, etc. (Baldí López \& García Quiroga 2005). En las ciudades, las psicologías colectivas (Freud) están ligadas al estrés, el congestionamiento, el suicidio, el hacinamiento, el egoísmo, la traición, la inseguridad y la desconfianza, lo cual genera personas mayores problemas de adicciones, trastornos, violencia, etc.; trastornos bañados del contexto y los medios de comunicación (Saxe Fernández 2005).

Tal como lo afirma Black (2013), para contrarrestar las situaciones vividas en las ciudades, en los cortos períodos de «ocio» las personas acuden desesperadamente a zonas con naturaleza como playas, montañas, lagos, etc., donde puedan olvidar y recuperarse al menos por unos cuantos días de la vida citadina, debido a que "(e)l enfoque instrumentalista 
del trabajo reduce al ser humano a una simple máquina productora de mercancías" (Quesada Monge 2015: 178).

La contaminación y degradación psicosocial en las grandes ciudades se enmarcan en la relación directa entre los humanos y su entorno, tanto así que "entre el 25 y el 30\% de la carga global por enfermedad ha sido estimado como atribuible al ambiente" (Baldí López \& García Quiroga 2005: 12). En el campo (en ocasiones) se tiene acceso al bienestar ambiental como aire limpio y tranquilidad, pero se carece de estilos de vida adecuados y condiciones socioeconómicas como acceso a salud, educación y otros que probablemente sean un poco más accesibles en las zonas más urbanas (al menos para algunos) (Lalonde).

\section{El caso de Caño Negro}

La región Huetar Norte permite analizar la cuestión del abandono de la tierra, y el gran esfuerzo que deben hacer quienes resisten. Hacia finales del siglo XIX la primera mitad del siglo XX, los huleros provenientes de Nicaragua, interesados en la extracción del árbol de hule (Castilla elastica), fueron desplazando las comunidades originarias de los Guatusos hacia el oeste (Escalera Reyes et al. 2013). Posteriormente también hubo otros acontecimientos históricos que, durante la segunda mitad del siglo XIX, generaron interés al incipiente Estado-nación costarricense por afirmar su soberanía territorial en las llanuras del norte, entre los cuales se puede citar la constante amenaza filibustera durante la Campaña Nacional de 1856 (Solórzano 2004).

Aún así, no fue hasta la Guerra Fría que el gobierno de Costa Rica, a través del Instituto de Tierras y Colonización (Itco), intervino la zona, debido a la necesidad de Estados Unidos de apoyar a la Contra nicaragüense para suprimir el movimiento sandinista que había logrado tomar el poder y realizar reformas después de varias décadas de régimen somocista (Escalera Reyes et al. 2013).

La gran violencia generada en Nicaragua ( $\mathrm{y}$ en el resto de Centroamérica), y posteriormente las reformas neoliberales, impulsó numerosas migraciones hacia Costa Rica, que terminaron por poblar la región Huetar Norte en los años setenta, ochenta y noventa. Los primeros colonos lograron apropiarse de gran cantidad de tierras para su subsistencia, debido 
a la gran extensión inexplorada. Fue precisamente en la década de los setenta cuando se oficializaron muchos de los poblados que actualmente existen y unos cuantos años después, la industrialización de la agricultura (Escalera Reyes et al. 2013).

La piña y los cítricos son los principales productos de exportación de esta región (Procomer, 2016), y su producción se encuentra concentrada principalmente bajo el dominio de grandes posesiones de tierra; en manos de poderosos capitales nacionales y transnacionales. Muchas de estas empresas han sido denunciadas por destrucciones ambientales y explotación social, las cuales sustentan su argumento principalmente en la generación de empleos, que les ha permitido incluso violar derechos humanos fundamentales (véase por ejemplo Morante 11-03-2016; Carazo \& Aravena 2016).

A pesar de la competencia desleal entre corporaciones y agricultores locales, el esquema del desarrollo agroindustrial creó expectativas, pero el tiempo ha demostrado que las comunidades no necesitan la instalación de grandes compañías, sino proyectos locales e integrales e incluso autónomos del sistema hegemónico. Quesada Webb (21-06-2017) asegura que para el caso de Matina, Los Chiles y Buenos Aires, más de la mitad del empleo es en el agro, y más de la mitad de los hogares tienen necesidades básicas insatisfechas. En muchos casos esto es alimentado a través de la exclusión que provoca el analfabestismo, que además facilita la explotación laboral mediante la contratación de "mano de obra poco calificada". Quesada Webb (21-06-2017) menciona que las personas dedicadas a la agricultura en el país tienen un promedio de edad de 53.9 años. Muchas de ellas deben trabajar de forma clandestina, migrar o soportar la explotación laboral.

Los datos del Centro Centroamericano de Población (CCP) en Los Chiles sobre no asistencia a educación formal y analfabetismo son alarmantes, sobre todo porque se continúa con el círculo vicioso de la no educación aprovechada por las compañías para contratar a las personas menos capacitadas (letradas) para explotarlas en labores físicas infrahumanas. El CCP (2017), contabiliza un número cercano a 9000 personas que no asisten a educación formal, y unas 2160 que sufren de analfabetismo; una tendencia que no se ha reducido desde 1984, año del que se tienen datos más antiguos. Este poco acceso a la educación se concibe como las difíciles condiciones que enfrentan las familias de la zona, las cuales, a pesar de en 
ocasiones acceder a becas o ayudas económicas (con largos, costosos y tediosos trámites) no logran cubrir los gastos.

Ante dichas limitaciones y riesgos de exponerse a trabajos forzados, nuestra postura alternativa es la agricultura de subsistencia, la pesca ${ }^{7}$ y el comercio local. En estas realidades, bajo los nuevos paradigmas de la psicología ambiental interesada en las correlaciones entre lo natural y lo antrópico, y en general el entorno en el que conviven las personas, la educación ambiental debe tener suma precaución en procurar mostrar un equilibrio entre el uso local no depredador- de recursos y la protección, pero nunca ver la utilización local de recursos como un acto criminal.

\section{1. ¿Protección o despojo? La conservación como deshumanización estratégica}

Al tocar el tema del uso local de recursos, es necesario tratar el asunto de la conservación en Caño Negro. En 1984, unas 9969 hectáreas fueron declaradas como el Refugio Nacional de Vida Silvestre Mixto Caño Negro. Este es uno de los sitios biológicamente más importantes del país, con grandes humedales que permiten la vida de especies. Es declarado desde 1991 sitio Ramsar por su importancia ecosistémica. Sin embargo, sus principales amenazas radican en la agricultura expansiva que se ha desarrollado en los alrededores (Acevedo Mairena \& Zamora Villalobos 2011).

En un principio, las comunidades locales tuvieron cierto acceso a los recursos ícticos de la zona, pero con el tiempo ese acceso ha sido cada vez más restringido (Escalera Reyes et al. 2013), ya que el fenómeno regional de conservación de la naturaleza y militarización supuestamente antinarco, pretende más bien tener controladas y deshumanizadas las áreas protegidas para investigarlas a través de estrategias como la bioprospección, para luego proceder con el (bio)saqueo de agua, flora, fauna, petróleo, gas, oro, etc., (Toly 2004; Delgado Ramos 2015), pasando por encima de las leyes nacionales y de los acuerdos ambientales (UICN 27-04-2017).

\footnotetext{
7. La pesca ha sido una práctica realizada por las poblaciones de Caño Negro, e incluso desde que los indígenas habitaban esta zona.
} 
Esta conservación absoluta y con cada vez más restricciones para los locales lleva nuevamente a la agroindustria, ya que esto ha llevado a las poblaciones a tener que someterse al trabajo forzado, ante la criminalización por incurrir sobre el Patrimonio Natural del Estado. Estas restricciones surgen por la supuesta preocupación por la conservación de la vida silvestre, ya que, claro está, siempre hay intereses por extraer más recursos de la cuenta. Sin embargo, el argumento de la conservación se cae por su propio peso cuando se observan los graves daños por la sedimentación y depósito de agrotóxicos que generan los monocultivos sobre los humedales protegidos (Carazo \& Aravena 2016).

Si verdaderamente las compañías piñeras fuesen sustentables, como lo asegura la WWF o Rainforest Alliance, ${ }^{8}$ e incluso el reciente informe de Incae (Guevara et al. 2017) que pretende justificar la expansión piñera en Osa, al sur del país, las destrucciones no serían tan perceptibles, y el repudio de las poblaciones no sería generalizado. El problema radica no solo en la destrucción ecológica, sino también en que las regiones donde establecen estas grandes compañías, son las regiones más empobrecidas, desiguales y con menor y decadente desarrollo humano del país (Estado de la Nación 2016).

\section{Volver a la tierra}

Saegert y Winkel (1990) establecen la relación del animal humano con el resto del ecosistema (en este caso natural), como una adaptación vital de la especie, que es determinante sobre el desarrollo psicológico (Baldí López \& García Quiroga 2005). Después de una creciente separación de lo antrópico y lo natural que ha conllevado a las más extremas destrucciones, durante la segunda mitad del siglo XX surge la preocupación desde diversas disciplinas de abordar aspectos ambientales (Baldí López \& García Quiroga 2005), ante las degradaciones ecológicas, sociales y económicas que estaba presentando el planeta y la humanidad, y que se siguen intensificando.

Bajo este inevitable contexto de colapso ecológico, social y económico (con extinciones en masa), en medio de un inexorable agotamiento de recursos, está provocando que sea cada vez más difícil alcanzar la equidad, la justicia y la solidaridad (Leff 2000). Se

\footnotetext{
${ }^{8}$. Estas organizaciones son certificadoras de calidad de las piñas costarricenses en Europa y Estados Unidos.
} 
trata de una lucha por la existencia y la competencia basada en la lógica capitalistaindividualista que predestina al ser humano como competitivo nato (Leakey \& Lewin 1995), descartando cualquier otra forma organizativa que no sea jerárquica o de apoyo mutuo (Kropotkin).

Un fenómeno latinoamericano e incluso mundial ha sido las revoluciones agroecológicas, que pretenden a través de la agroecología en sus tres dimensiones (cognitiva, tecnológica y social) que conforman la revolución, en conjunto con otros conocimientos como la filosofía, la sociología y el arte, para crear alternativas al sistema depredador (de recursos y mano de obra) actual (Artieri \& Toledo 2010).

Los principios básicos de la agroecología incluyen: el reciclaje de nutrientes y energía, la sustitución de insumos externos; el mejoramiento de la materia orgánica y la actividad biológica del suelo; la diversificación de las especies de plantas y los recursos genéticos de los agroecosistemas en tiempo y espacio; la integración de cultivos con ganadería, y la optimización de las interacciones y la productividad del sistema agrícola en su totalidad, en lugar de los rendimientos aislados de las distintas especies (Artieri \& Toledo 2010: 165).

Este planteamiento consiste en volver a ver en la tierra formas de subsistencia en medio del modelo agroindustrial, tomado como base primordial la organización comunitaria y el intercambio comercial y de conocimientos a nivel local. Estudios en México y Guatemala han demostrado que una hectárea de tierra utilizada mediante prácticas agroecológicas es suficiente para brindar las calorías necesarias de maíz para una familia de entre cinco y siete personas (Wilken 1987).

Asimismo, es importante acudir a nociones holísticas como la Permacultura y sus tres principios éticos básicos desarrollados por David Holmgren: 1) cuidar a las demás personas, 2) cuidar a la naturaleza, y 3) los productos y excedentes redistribuirlos en cuidar a las personas y cuidar a la naturaleza (Holmgren 2013). En ese sentido, la organización se vuelve fundamental para enfrentar el colapso climático antropogénico como uno de los principales retos en la agricultura acudiendo a técnicas agroecológicas.

\subsection{La experiencia en El Sitio y Las Flores}

El Sitio y Las Flores es una comunidad producto de un actual proceso de recuperación de tierras, que posee una población de 62 habitantes. Al igual que otras comunidades de la 
región Huetar Norte, sufren la explotación laboral y las destrucciones ecosociales de la industria agrícola. Una parte de la población (32\%) es de origen nicaragüense, proveniente, como ya se mencionó, producto de las guerras. En El Sitio y Las Flores, el 73\% de la población no tiene la primaria completa o no tienen ninguna base escolar de lo que se deriva que un $8 \%$ de la población no sepa leer ni escribir, incidiendo indiscutiblemente en que estos no puedan optar por mejores oportunidades laborales (observación personal).

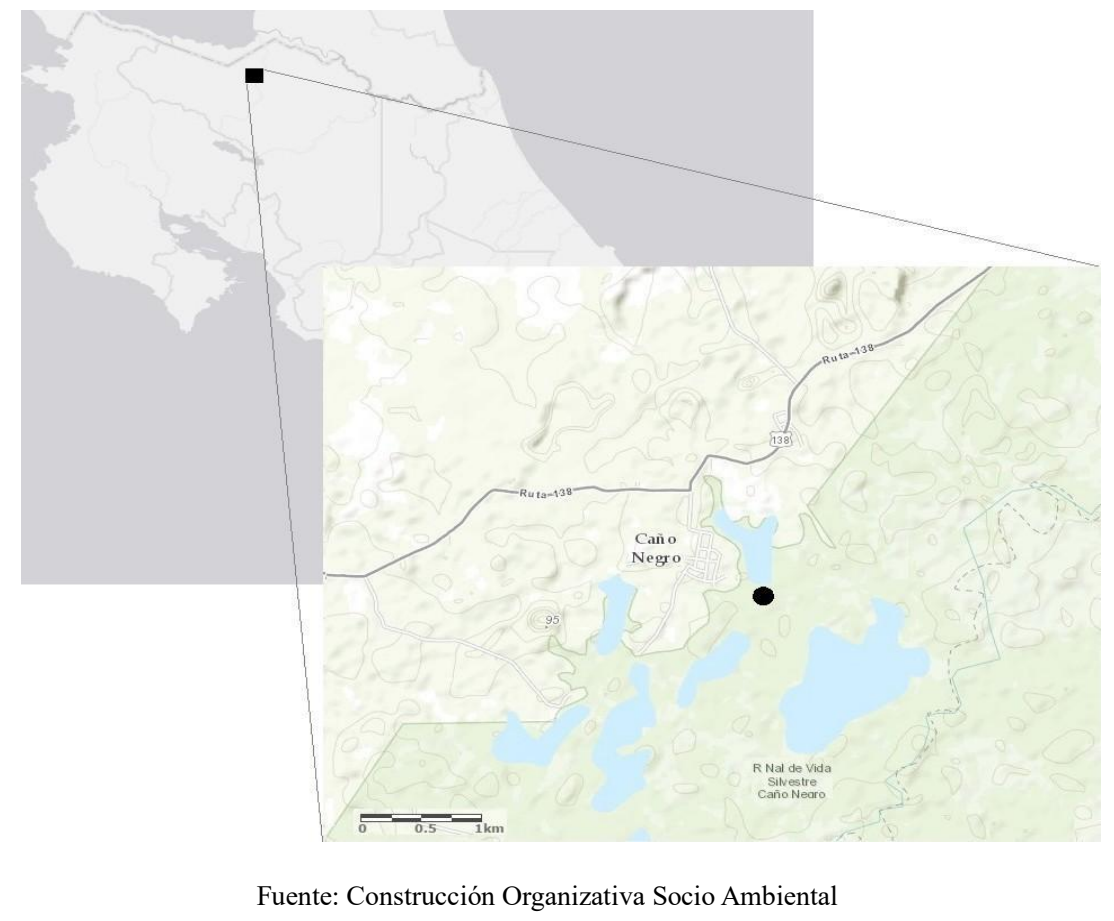

FIGURA 1. Ubicación de El Sitio y Las Flores, Caño Negro, Costa Rica

Sus principales actividades de subsistencia son el trabajo en los monocultivos, la agricultura y pesca de subsistencia. La tenencia de tierra se plasma en estas dos comunidades como un aspecto fundamental, ya que permite producir alimentos sin necesidad de comprarlos. Otras formas de recaudación de ingresos económicos recientemente han sido posibles gracias a la organización comunitaria, mediante actividades festivas como ferias, partidos de fútbol y demás, que dinamizan la economía local más allá de la necesidad económica que impulsa el trabajo forzado.

Partiendo de un acercamiento externo a la comunidad, es claro que el objetivo central de cualquier proceso de organización comunitaria debe ser que la participación de los agentes externos deje de ser necesaria, y que las acciones de intervención a los entornos sociales y 
espaciales por parte de la comunidad se lleven a cabo impulsados por las propias dinámicas endógenas. Con lo anterior, queremos decir que cuando la organización comunitaria alcanza niveles de organización que permitan la continuidad de los objetivos establecidos, es preciso que los agentes externos se hagan a un lado.

El colectivo estudiantil Cosa llegó a esta zona en 2015 a través del apoyo del programa Iniciativas Estudiantiles de la Vicerrectoría de Acción Social (Vas) de la Universidad de Costa Rica (UCR), planteando una metodología que fuese capaz de adaptarse a las necesidades y deseos de las comunidades, y también que fuese capaz de tener un amplio alcance en cuanto a interdisciplinariedad. Para eso, se ha acudido a disciplinas como biología, ciencias del deporte, agronomía, sociología, historia, educación (no formal) ${ }^{9}$, sexualidad, arquitectura, bioconstrucción, agroecología, psicología y otras.

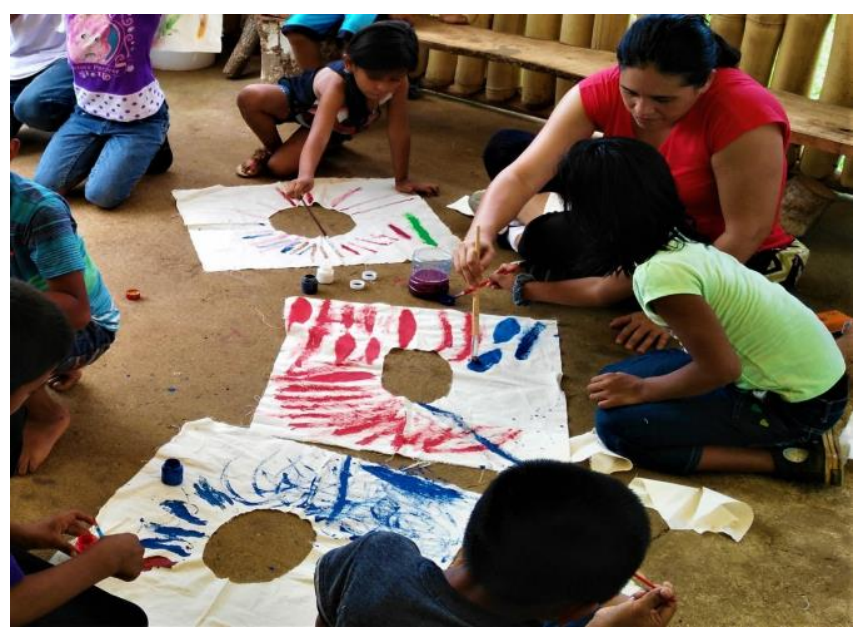

Fuente: Construcción Organizativa Socio Ambiental

FIGURA 2. Actividad psicológica con niños y niñas de El Sitio y Las Flores, Caño Negro, Costa Rica

Para este caso, la actividad con apoyo psicológico de Adriana Ospina Vélez a través de la coordinadora de Iniciativas Estudiantiles, Mariana Buzó Garay, ha impulsado un trabajo fundamental con la niñez, en el que, como el caso de la figura, los y las niñas dibujaron y escribieron cuentos. También, el proceso de investigación y acción que se ha llevado a cabo

\footnotetext{
${ }^{9}$. La educación no formal se vuelve fundamental ante las realidades de estas comunidades, las cuales muchas veces poseen pocas posibilidades socioeconómicas adversas para letrar a sus pobladores (niños, jóvenes y adultos). Por lo tanto, la educación no formal responde a esas necesidades, siendo una forma inclusiva de aprender, también de forma lúdica.
} 
en la zona Norte-Norte ha permitido generar una contextualización socioeconómica y ambiental de la región, esto a través del mapeo de actores, diagnósticos participativos y la consolidación de una base organizativa; así mismo, al estudio de cotidianidades, problemáticas y eventos históricos que han sido abordados desde un enfoque multidisciplinario que respalda el trabajo en comunidad y retroalimenta la recopilación de datos para la investigación.

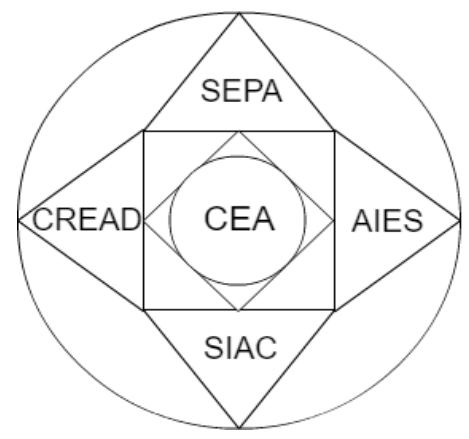

Fuente: Construcción Organizativa Socio Ambiental

FIGURA 3. Metodología "Mandala de Cosa" implementada en El Sitio y Las Flores, Caño Negro, Costa Rica

Este proceso de acompañamiento surge de la propuesta metodológica que toma como aspecto central el interés de crear Comunidades Ecosostenibles y Autosustentables (CEA) en los distintos aspectos de la cotidianeidad. Como ejes temáticos que se desprenden de este núcleo, se encuentran las nociones de:

- $\quad$ Construir, Renovar, la Educación con Arte y Deporte (CREAD) que promueve el movimiento, el ocio y los diversos talentos, además de la educación informal,

- $\quad$ Salud Integral con Alternativas Comunitarias (SIAC) para promover la salud comunitaria y el cuido de las personas,

- $\quad$ Sistema Espacial de Producción Autónoma (SEPA), ligado a la soberanía alimentaria y,

- $\quad$ Área de Infraestructuras Espaciales Sostenibles (AIES) enfocado en la parte infraestructural. 
El trabajo comunitario se ha dividido en cuatro fases:

\subsubsection{Diagnóstico y fortalecimiento de organización comunitaria}

Esta etapa que abarcó de noviembre de 2014 a mayo de 2015 comenzó con la proposición a la comunidad de entablar una relación de cooperación, en la cual el colectivo Cosa acompañó el proceso organizativo y facilitó mediaciones y herramientas que coadyuvaron dicho proceso. Se trabajó, a través de talleres participativos y dinámicas lúdicas, en el mejoramiento de la comunicación intracomunitaria y la reflexión sobre los alcances y límites de la organización comunitaria. El objetivo central fue el establecimiento de un nivel mínimo de tejido organizativo que permitiera la eventual realización de acciones tangibles en la comunidad.

\subsubsection{Puesto en práctica}

Se identificó, de mayo a diciembre de 2015, la necesidad de contar con un espacio comunitario para reuniones, capacitaciones y demás actividades. La comunidad por cuenta propia comenzó la construcción de un Rancho Comunitario y Cosa facilitó la generación de una alianza entre la comunidad y el colectivo Semillas ${ }^{10}$, quienes acompañaron el proceso de diseño participativo del rancho y construcción.

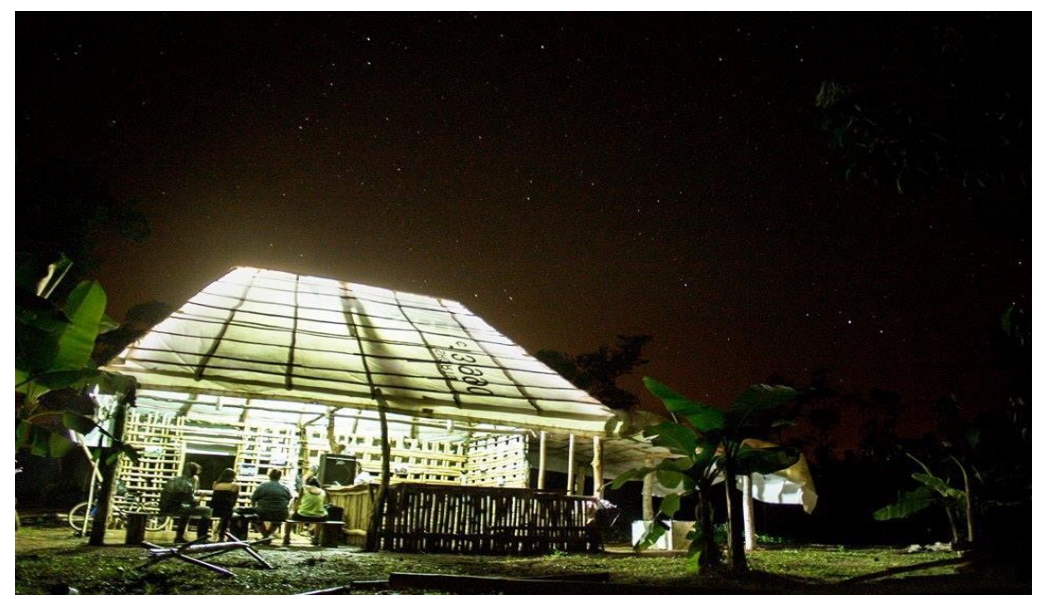

Fuente: Construcción Organizativa Socio Ambiental

FIGURA 4. Rancho comunitario de El Sitio y Las Flores, Caño Negro, Costa Rica

${ }^{10}$. Semillas es un colectivo interdisciplinario que impulsa la arquitectura social comunitaria para mejorar la calidad de los espacios y hábitats humanos a través de la articulación intersectorial y la cogestión de procesos participativos. 
El proceso constructivo generó dinámicas comunitarias que fortalecieron al tejido organizativo. Además, se trabajó en conjunto con el colectivo Pausa Urbana ${ }^{11}$ en la instalación y utilización de un bicigenerador eléctrico (Pausa Urbana 2016). El rancho fue inaugurado en diciembre de 2015 con una Feria Comunitaria. Este espacio también ha funcionado para la proyección de materiales audiovisuales y en general para la realización de actividades en torno a la educación no formal, agricultura, arte, organización, etc.

Estos procesos de reflexión-acción-reflexión, no se ha basado en enseñar, sino en acompañar a través del involucramiento con la comunidad y la realización de actividades prácticas, ya que la "realización exitosa de tareas, por pequeñas que sean, produce el placer de haber realizado bien algo, de alcanzar una meta que de alguna manera modifica una situación que se desea distinta" (Montero 2003: 82).

\subsubsection{Creando espacios de organización e intervención a través del Rancho}

\section{Comunitario}

De nada sirve un espacio infraestructural si no se le da contenido comunitario. Durante 2016 se planteó continuar con la cooperación de parte de Iniciativas Estudiantiles, a través de un proyecto que pretendía trabajar los siguientes ejes: Economía social, Huerta comunitaria y Educación popular. Se construyó un horno envolvente y se inició la elaboración de una huerta comunitaria gracias a la colaboración de la Caravana Mesoamericana para el Buen Vivir. ${ }^{12}$

\footnotetext{
11. Pausa Urbana es una fundación transdisciplinaria conformada por profesionales y estudiantes de arquitectura, artes, ciencias sociales y derecho; además de una amplia red de artistas y colectivos afines con quienes se trabaja de acuerdo a la naturaleza de cada proyecto, con el objetivo de buscar e implementar de formas alternativas de gestión urbana desde la gente, a través del uso, la apropiación sana e inclusiva y la activación de espacios públicos para convertirlos en verdaderos ámbitos de convivencia para todos y todas. ${ }^{12}$. Es un grupo de jóvenes que ha viajado por Mesoamérica para compartir experiencias del buen vivir. Algunos movimientos policiacos han intentado reprimirlos en Nicaragua y México. Actualmente se encuentran en la preparación de un libro que llevará por nombre "Mesoamérica se Levanta: un libro desde las resistencias para las resistencias".
} 


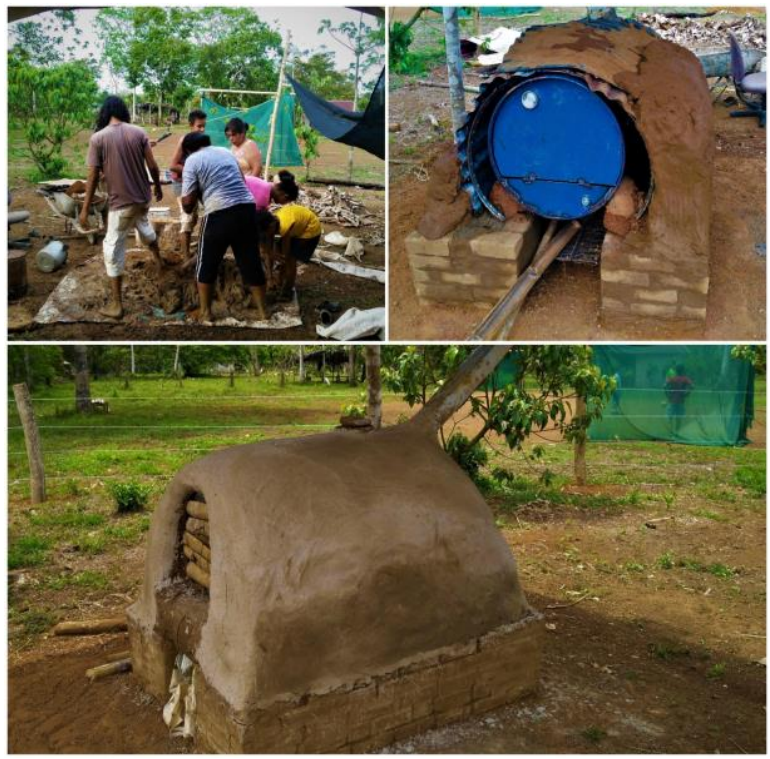

Fuente: Construcción Organizativa Socio Ambiental

FIGURA 5. Horno construido con barro en El Sitio y Las Flores, Caño Negro, Costa Rica

La organización tomó su propio ritmo, comenzaron a organizar actividades sin que tuviera que mediar nuestra participación. Ejemplos de lo anterior son la intervención a la estructura y alrededores del Rancho Comunitario, además de la organización de Ferias Comunitarias que devengaron recursos económicos. Por lo tanto, se consideró que era necesario cerrar nuestro ciclo en la comunidad, y se les planteó que a partir de 2017 se dejaría de participar con el mismo rol y frecuencia en los procesos de la comunidad.

\subsubsection{Coyuntura posthuracán}

El paso del huracán incidió negativamente en el proceso organizativo. Se generaron rencillas y un relativo debilitamiento de la estructura organizativa. Además, quedó de manifiesto la gran vulnerabilidad que padece la comunidad.

El riesgo se origina en función de la presencia de dos elementos: la amenaza y la vulnerabilidad. Al ser la sociedad incapaz de determinar de forma total el fluctuar del entorno natural, se puede afirmar que siempre habrá amenazas latentes; sin embargo, la prevención y preparación pueden definir el nivel de afectación que una comunidad recibirá a raíz de la presencia de una amenaza natural. En cuanto al caso de El Sitio y Las Flores, su nivel de 
vulnerabilidad radica en tres grandes aspectos: la cercanía con el río Frío, la precaria estructura material de las viviendas y la ausencia de acciones tendientes a la prevención de eventuales amenazas naturales.

Nuestro ciclo en la comunidad se pretendía cerrar con el denominado Festival Cultural del Guajipal; sin embargo, tuvo que ser cancelado ante la llegada del huracán. Por fortuna, no hubo vidas humanas que lamentar, no obstante, sí hubo considerables daños materiales.

\subsubsection{Acompañamiento hacia la gestión del riesgo}

Lamentablemente, la institucionalidad nacional ha preponderado el enfoque de atención de emergencias por encima de la gestión integral del riesgo. El Sistema Nacional de Prevención de Riesgo ofrece una herramienta de gran utilidad pero poco utilizada; los Comités Comunales de Emergencias. Estos comités comunales no funcionaron durante el advenimiento del Huracán Otto. Además, los pocos existentes padecen de problemáticas relaciones con los comités municipales y regionales.

Ante esta situación, entre los proyectos en desarrollo en esta nueva etapa de Cosa, se encuentra la conformación del Comité Comunal de Emergencias, el cual está siendo elaborado a través de un diagnóstico y especificación participativa de las propuestas de acción. Además, la gestión del riesgo, nos lleva nuevamente hacia la necesidad de la autonomía alimentaria en un marco de colectividad. Durante las emergencias, las vías de comunicación, y en general el comercio se paraliza, y es necesario contar con planes estratégicos que permitan a las personas seguir teniendo acceso a la alimentación. Como estos eventos naturales son generalmente climáticos, la idea de la construcción de un invernadero comunal, el cual funcione de modelo para la construcción de otros a escalas familiares, se encuentra entre los planes de la gestión del riesgo.

Por tanto, estos procesos, los cuales son atravesados por la organización comunitaria en todos sus espacios, y que son alimentados por nociones como la autonomía alimentaria colectiva, la economía solidaria, la educación popular, y más reciente con la gestión del riesgo, representan procesos fundamentales no solo para enfrentar las dificultades históricas de la región, sino también para crear espacios en los que se pueda alcanzar la dignidad más 
allá del intento de acumulación de capital, sobre todo en un contexto en el que los recursos fundamentales- se están agotando cada vez más sin posibilidad alguna de retorno.

\section{Conclusiones}

Ante la realidades ecosociales que atraviesa la humanidad, la autonomía alimentaria -al menos parcial y colectiva- se vuelve una alternativa de resistencia ante las adversas desigualdades y carencias. Para eso, es necesario voltear la mirada a las resistencias y formas de subsistencia de los pueblos indígenas y campesinos sobre todo en América Latina.

La implementación de conocimientos desde diversas áreas a través de la educación no formal, además del acompañamiento por parte de organizaciones, colectivos, etc., pretende -y debe- enrumbarse a la subsistencia en medio del capitalismo neoliberal, por medio de las organizaciones comunales, la economía solidaria, y en fin, la mejora de las condiciones de vida.

La búsqueda de una autonomía contrahegemónica, la organización y la recuperación de prácticas de subsistencia de las comunidades representa un brazo de las resistencias al sistema de enclave del extractivismo de los monocultivos piñeros, naranjeros, palmeros, cañeros, etc. El otro brazo de resistencia se encuentra en la lucha de presión en instituciones estatales e internacionales para mejorar las situaciones actuales.

Esto se vuelve necesario porque más allá de un actor indiferente, el Estado se ha convertido en el principal enemigo de las poblaciones campesinas, al legitimar la depredación

de recursos, las condiciones sociales y laborales adversas, la acumulación de la tierra y la expulsión campesina, utilizando la instumentalización laboral como base para la creación del argumento de la generación de empleo. 


\section{Referencias}

Acevedo Mairena, Heiner \& Zamora Villalobos, Mauricio. (2011). Refugio Nacional de Vida Silvestre Mixto Caño Negro: Plan de Manejo 2011-2020. San José: Minae-Sinac- INBio.

Alonso Reynoso, Carlos \& Alonso Sánchez, Jorge. (2015). En busca de la libertad de los de abajo: La demoeleuthería. Jalisco: Editorial CUCSH-UDEG.

Altieri, Miguel \& Toledo, Víctor Manuel. (2010). La revolución agroecológica de América Latina: Rescatar la naturaleza, asegurar la soberanía alimentaria y empoderar al campesino. El Otro Derecho 42: 163-202.

Araya, Jorge. (03-06-2015). Costa Rica es el consumidor más voraz de plaguicidas en el mundo. Semanario Universidad. [Fecha de último acceso 21-03-2018]. $<\underline{\text { https://goo.gl/D88p7Y }>\text {. }}$

Baldí López, Graciela \& García Quiroga, Eleonora. (2005). Calidad de vida y medio ambiente. La psicología ambiental. Universidades 30: 09-16.

Black, Bob. (2013). La abolición del trabajo. Logroño: Pepitas de Calabaza.

Bookchin, Murray. (1999). La ecología de la libertad. El surgimiento y la disolución de la jerarquía. Madrid: Nossa y Jara Editores.

Carazo, Eva \& Aravena, Javiera. (2016). Condiciones de producción, impactos humanos y ambientales en el sector piña en Costa Rica. San José: Oxfam.

CCP (Centro Centroamericano de Población). (2017). Infocensos. Centro Centroamericano de Población. [Fecha de último acceso 20-06-2017]. < https://goo.gl/5dVbJD $>$.

Comité Invisible. (2015). A nuestros amigos. Logroño: Pepitas de Calabaza.

Consejo de Derechos Humanos. (2017). Informe de la Relatora Especial sobre el derecho a la alimentación. Ginebra: Naciones Unidas. 
Córdoba, Javier \& Rodríguez, Alina. (03-06-2015). Del Monte es el sujeto privado con más concesiones de agua en el país. Semanario Universidad [Fecha de último acceso 15-052017]. <https://goo.gl/a4XPDi $>$.

Delgado Ramos, Giancarlo. (2011). El mito de la economía verde. Ambientico 219: 28-39.

Delgado Ramos, Giancarlo. (2015). Biodiversidad, desarrollo sustentable y militarización: Esquemas del saqueo en Mesoamérica. México DF: Centro de Investigaciones Interdisciplinarias en Ciencias y Humanidades-Unam.

Escalera Reyes, Javier; Cáceres, Rafael \& Díaz Aguilar, Antonio. (2013). "Las apariencias engañan". Conservación, sociedad local y relaciones de poder: El caso de Caño Negro (Costa Rica). Revista de Antropología Iberoamericana 8(3): 369-394.

Estado de la Nación. (2016). Vigésimosegundo informe estado de la nación en desarrollo humano sostenible. San José: Programa Estado de la Nación.

Fao (Organización de las Naciones Unidas para la Alimentación y la Agricultura). (2015). Estado de la inseguridad alimentaria en el mundo 2015. Roma: Fao.

Farah, Ivonne \& Vasapollo, Luciano. (2011). Vivir bien: ¿Paradigma no capitalista? La Paz: Cides-Umsa.

Global Witness. (2016). En Terreno Peligroso. London: Global Witness.

Guereña, Arantxa. (2016). Desterrados. Tierra, poder y desigualdad en América Latina. Washington: Oxfam.

Guevara, Andrés; Arce, Ronald \& Guevara, Porfirio. (2017). Impacto económico, social y ambiental de la piña en Costa Rica. San José: Incae.

Holmgren, David. (2013). Permacultura. Principios y senderos más allá de la sustentabilidad. Castellón: Kaicron.

Leakey, Richard \& Lewin, Roger. (1995). La sexta extinción. El futuro de la vida y de la humanidad. Barcelona: Turquets Editores. 
Leff, Enrique. (Coord.). (2000). La complejidad ambiental. México DF: Siglo XXI Editores. Llaguno Thomas, José Julián; Cerdas Vega, Gerardo \& Aguilar Sánchez, Carlos. Transformaciones y continuidades en el capitalismo agrario centroamericano: el caso de Costa Rica. En: Almeyra, Guillermo; Concheiro Bórquez, Luciano; Mendes Pereira, João Márcio \& Porto-Gonçalves, Carlos Walter. (2012). (coords.). Capitalismo: Tierra y poder en América latina (1982-2012). Costa Rica, Cuba, El Salvador, Guatemala, Honduras, México, Nicaragua. Volumen III. Buenos Aires: Ediciones Continente.

Montero, Maritza. (2006). Teoría y práctica de la psicología comunitaria. Buenos Aires: Editorial Paidós.

Morante, Ángela. (11-03-2016). Trabajadores de Ticofrut denuncian seguridad armada en empresa para evitar intento de huelga. Elmundo.cr. [Fecha de último acceso 15-05-2017]. $<\underline{\text { https://goo.gl/9OEqko }>\text {. }}$

Pausa Urbana. (2016). Bailando con el planeta_Caño Negro. Pausa Urbana. [Fecha de último acceso 20-06-2017]. <https://goo.gl/ciLv63>.

Petras, James \& Veltmeyer, Henry. (2002). Los campesinos y el Estado en América Latina: Un pasado turbulento, un futuro incierto. Problemas del Desarrollo 33(131): 7-64.

Procomer (Promotora de Comercio Exterior de Costa Rica). (2016). Estadísticas de Comercio Exterior. Costa Rica 2016. Exportaciones e importaciones. Procomer. [Fecha de último acceso 26-01-2018].<https://goo.gl/jx8vph>.

Quesada Monge, Rodrigo. (2014). Anarquía. Orden sin autoridad. Heredia: Euna-Editorial Eleuterio

Quesada Webb, Armando. (21-06-2017). La Agricultura: un oficio sin herederos. Semanario

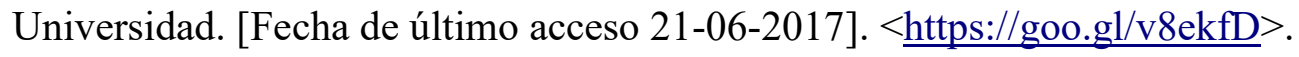

Rosset, Peter. (2009). Food Sovereignty in Latin America: Confronting the 'New' Crisis. NACLA Report on the Americas: 16-21. 
Saegert, Susan \& Winkel, Gary. (1990). Environmental Psychology. Annual Review of Psychology 41(1): 441-477. DOI: 10.1146/annurev.ps.41.020190.002301

Saxe Fernández, Eduardo. (2005). Colapso mundial y guerra. San José: Amo al Sur Editorial.

Solórzano, William. (2004). La colonización agrícola de la Región Huetar Norte de Costa Rica 1884-1995. Tesis para optar por el grado de Magister en el Posgrado de Historia UCR.

Toledo, Víctor Manuel; Boege, Eckart \& Barrera Bassols, Narciso. (2010). The biocultural heritage of México: an overview. Landscape 3: 6-10.

Toly, Noah. (2004). Globalization and the Capitalization of Nature: A Political Ecology of Biodiversity in Mesoamerica. Bulletin of Science, Technology \& Society 24(1): 47-54.

UICN (Unión Internacional para la Conservación de la Naturaleza) (27-04-2017). La condición de Patrimonio Mundial no disipa las amenazas de la minería y las represas en sitios naturales emblemáticos. UICN. [Fecha de último acceso 15-05-2017]. $<\underline{\text { https://goo.gl/DbKfNk}>}$.

United Nations. (2013). Wake up before it is too late: Make agriculture truly sustainable now for food security in a changing climate. Ginebra: United Nations.

Wilken, Gene. (1987). Good farmers: traditional agricultural resource management in Mexico and Guatemala. Berkeley: University of California Press.

\section{(a) $(\mathbb{Q} \Theta$}

